\title{
High-precision search for dark photon dark matter with the Parkes Pulsar Timing Array
}

Xiao Xue, ${ }^{1}$ Zi-Qing Xia, ${ }^{2}$ Xingjiang Zhu, ${ }^{3,4,5}$ Yue Zhao, ${ }^{6}$ Jing Shu $\odot,{ }^{7,8,9,10,11,12,{ }^{*}}$ Qiang Yuan, ${ }^{2,10,13, \dagger}$ N. D. Ramesh Bhat, ${ }^{14}$ Andrew D. Cameron, ${ }^{5,15,16}$ Shi Dai, ${ }^{17,15}$ Yi Feng, ${ }^{18}$ Boris Goncharov, ${ }^{4,5}$ George Hobbs, ${ }^{15}$ Eric Howard, ${ }^{15,19}$

Richard N. Manchester, ${ }^{15}$ Aditya Parthasarathy, ${ }^{16,20}$ Daniel J. Reardon, ${ }^{5,16}$ Christopher J. Russell, ${ }^{21}$ Ryan M. Shannon, ${ }^{5,16}$ Renée Spiewak, ${ }^{16,22}$ Nithyanandan Thyagarajan, ${ }^{23}$ Jingbo Wang, ${ }^{24}$ Lei Zhang, ${ }^{25}$ and Songbo Zhang ${ }^{26}$

(PPTA Collaboration)

${ }^{1}$ II. Institut für Theoretische Physik, Universität Hamburg, Luruper Chaussee 149, D-22761 Hamburg, Germany

${ }^{2}$ Key Laboratory of Dark Matter and Space Astronomy, Purple Mountain Observatory, Chinese Academy of Sciences, Nanjing 210023, China

${ }^{3}$ Advanced Institute of Natural Sciences, Beijing Normal University at Zhuhai 519087, China

${ }^{4}$ School of Physics and Astronomy, Monash University, Clayton, Victoria 3800, Australia

${ }^{5}$ OzGrav: The ARC Centre of Excellence for Gravitational Wave Discovery, Hawthorn, Victoria 3122, Australia

${ }^{6}$ Department of Physics and Astronomy, University of Utah, Salt Lake City, Utah 84112, USA

${ }^{7}$ CAS Key Laboratory of Theoretical Physics, Institute of Theoretical Physics, Chinese Academy of Sciences, Beijing 100190, China

${ }^{8}$ School of Physical Sciences, University of Chinese Academy of Sciences, Beijing 100049, China

${ }^{9}$ CAS Center for Excellence in Particle Physics, Beijing 100049, China

${ }^{10}$ Center for High Energy Physics, Peking University, Beijing 100871, China

${ }^{11}$ School of Fundamental Physics and Mathematical Sciences, Hangzhou Institute for Advanced Study,

University of Chinese Academy of Sciences, Hangzhou 310024, China

${ }^{12}$ International Centre for Theoretical Physics Asia-Pacific, Beijing/Hangzhou, China

${ }^{13}$ School of Astronomy and Space Science, University of Science and Technology of China, Hefei 230026, China

${ }^{14}$ International Centre for Radio Astronomy Research, Curtin University, Bentley, Western Australia 6102, Australia

${ }^{15}$ Australia Telescope National Facility, CSIRO Astronomy and Space Science, Epping, New South Wales 1710, Australia

${ }^{16}$ Centre for Astrophysics and Supercomputing, Swinburne University of Technology, Hawthorn, Victoria 3122, Australia

${ }^{17}$ School of Science, Western Sydney University, Locked Bag 1797, Penrith South DC, NSW 2751, Australia

${ }^{18}$ Research Institute of Artificial Intelligence, Zhejiang Lab, Hangzhou, Zhejiang 311121, China

${ }^{19}$ Macquarie University, Department of Physics and Astronomy, Sydney, New South Wales 2109, Australia

${ }^{20}$ Max-Planck-Institut für Radioastronomie, Auf dem Hügel 69, D-53121 Bonn, Germany

${ }^{21}$ CSIRO Scientific Computing, Australian Technology Park, Alexandria, New South Wales 1435, Australia

${ }^{22}$ Jodrell Bank Centre for Astrophysics, University of Manchester, Manchester M13 9PL, United Kingdom

${ }^{23}$ National Radio Astronomy Observatory, Socorro, New Mexico 87801, USA

${ }^{24}$ Xinjiang Astronomical Observatory, Chinese Academy of Sciences, Urumqi, Xinjiang 830011, China

${ }^{25}$ School of Physics and Technology, Wuhan University, Wuhan 430072, China

${ }^{26}$ Purple Mountain Observatory, Chinese Academy of Sciences, Nanjing 210023, China

(Received 1 December 2020; revised 8 June 2021; accepted 10 December 2021; published 22 February 2022)

The nature of dark matter remains obscure in spite of decades of experimental efforts. The mass of dark matter candidates can span a wide range, and its coupling with the Standard Model sector remains uncertain. All these unknowns make the detection of dark matter extremely challenging. Ultralight dark matter, with $m \sim 10^{-22} \mathrm{eV}$, is proposed to reconcile the disagreements between observations and predictions from simulations of small-scale structures in the cold dark matter paradigm while remaining consistent with other observations. Because of its large de Broglie wavelength and large local occupation number within galaxies, ultralight dark matter behaves like a coherently oscillating background field with an oscillating frequency dependent on its mass. If the dark matter particle is a spin-1 dark photon, such as the $U(1)_{B}$ or $U(1)_{B-L}$ gauge boson, it can induce an external oscillating force and lead to displacements of test masses. Such an effect would be observable in the form of periodic variations in the arrival times of radio pulses from highly stable millisecond pulsars. In this study, we search for evidence of ultralight dark photon dark matter (DPDM) using 14-year high-precision observations of 26 pulsars collected with the Parkes Pulsar Timing Array. While no statistically significant signal is found, we place constraints on coupling constants for the $U(1)_{B}$ and $U(1)_{B-L}$ DPDM. Compared with other experiments,

\footnotetext{
*Corresponding author: jshu@itp.ac.cn
}

†Corresponding author: yuanq@pmo.ac.cn

Published by the American Physical Society under the terms of the Creative Commons Attribution 4.0 International license. Further distribution of this work must maintain attribution to the author(s) and the published article's title, journal citation, and DOI. 
the limits on the dimensionless coupling constant $\epsilon$ achieved in our study are improved by up to two orders of magnitude when the dark photon mass is smaller than $3 \times 10^{-22} \mathrm{eV}\left(10^{-22} \mathrm{eV}\right)$ for the $U(1)_{B}\left(U(1)_{B-L}\right)$ scenario.

DOI: 10.1103/PhysRevResearch.4.L012022

Introduction. About $26 \%$ of the total energy in our presentday Universe is composed of dark matter. The cold dark matter paradigm has been widely accepted since it explains most of the cosmological observations over a large span of redshift [1]. However, numerical simulations of the traditional particle like cold dark matter models show that the central density profile of dark matter in dwarf galaxies is much steeper than that inferred from observed rotation curves, and the number of satellite galaxies of Milky Way-like hosts predicted from simulations is higher than that inferred from observations [2]. These so-called "core-cusp problem" and "missing-satellite problem" impose challenges to the the conventional cold dark matter paradigm. Baryonic feedback effects [3] may be a viable solution, but it is a nontrivial task. Meanwhile, various alternative dark matter models have been proposed to address such small-scale shortcomings, e.g., warm dark matter [4], self-interacting dark matter [5], and fuzzy dark matter [6].

A dark photon is a hypothetical particle from the theory beyond the Standard Model of particle physics. Just like the ordinary photon, a dark photon is the gauge boson of a $U(1)$ interaction. The existence of the dark photon is well predicted in many string-inspired models, such as large-volume string compactifications [7-9]. The dark photon mass can be generated by either the Higgs mechanism or the Stueckelberg mechanism, and it is naturally light [10]. When this $U(1)$ symmetry is the baryon or lepton number or their linear combination, protons, neutrons, and electrons are "charged" under this symmetry, and there will be an extra force (i.e., the "fifth force") between objects that consist of ordinary matter.

In recent years, the dark photon has been widely considered as a viable dark matter candidate. Interestingly, when the dark photon is ultralight $\left(m_{A} \sim 10^{-22} \mathrm{eV}\right)$, which represents a realization of the fuzzy dark matter paradigm, its de Broglie wavelength is up to subgalactic scale, i.e., $O(0.1-1) \mathrm{kpc}$, and the occupation number in dark matter halos is very large [11]. These aspects imply that the "wave nature" of dark matter particles is pronounced; hence the dark matter can be properly treated as an oscillating background field rather than individual particles. Because of the formation of a "soliton core" instead of a density cusp at the center of galaxies [12-14], the density of the dark matter halo at a galactic center becomes flat. This provides a better fit to observations than a cuspy Navarro-Frenk-White profile [15] predicted in the cold dark matter scenario. In addition, the substructures formed under the fuzzy dark matter hypothesis are fragile against tidal disruptions, since they usually have less concentrated mass distributions. This solves the "missing-satellite" problem [16] at the same time. To date, the ultralight dark photon dark matter (DPDM) leads to predictions consistent with most existing observations at various scales [16], making it one of the most compelling dark matter candidates.

Conventional direct detection experiments [17-19] are not sensitive to fuzzy dark matter particles because of the ex- tremely small energy and momentum depositions in elastic scatterings. If the ultralight dark matter particle is a $U(1)_{B}$ or $U(1)_{B-L}$ gauge boson (i.e., dark photon), the fifth-force experiments can be used to put stringent constraints on the existence of such particle [20]. Furthermore, the dark matter background can cause displacements on terrestrial/celestial objects, leading to observable effects [21]. For example, depending on the mass of dark photon particles which determines the dark matter oscillation frequency, such motion can be probed using high-precision astrometry [22], spectroscopic [23], timing observations [24,25], as well as gravitational-wave detectors [26-28].

Among all these relevant observations, the pulsar timing array (PTA) experiments are of particular interest to us. Millisecond pulsars with very high rotational stability are observed to emit periodic electromagnetic pulses with incredible accuracy. For the best pulsars, the measurement uncertainties of pulse arriving times are below $100 \mathrm{~ns}$. The information of possible new physics (including signatures of DPDM) is in the irregularities of pulse arrival times, which are usually called timing residuals. The $U(1)_{B}$ or $U(1)_{B-L}$ DPDM with an ultralight mass $\sim 10^{-22} \mathrm{eV}$, induces displacements of Earth and pulsar that cause a periodic signal in timing residuals with frequency [29] $f=m_{A} / 2 \pi \approx 2.4 \times 10^{-8} \mathrm{~Hz}$. This frequency falls into the sensitive region of PTA experiments, which have collected measurements of pulse arrival times on weekly to monthly cadence over time scales of 10-20 years.

In this work, we make use of the second public data release of the Parkes PTA project $[31,32]$, to search for evidence of the DPDM. Subsets of these data have been used to search for stochastic gravitational waves [33] as well as the gravitational effects induced by ultralight scalar dark matter [24]. Compared with Ref. [24], we discuss the direct coupling between DPDM and the ordinary matter in this work, which results in different signals from the gravitational effect studied in Ref. [24]. We also improve the analysis through considering the correlation of pulsars in the DPDM field, the interference of DPDM wave functions, and excluding fake signals that arise from the time-frequency method. (see Secs. I and II of the Supplemental Material [34] for details.)

Observations. The second data release (DR2 [35]) of the PPTA project [32] includes high-precision timing observations for 26 pulsars collected with the 64-m Parkes radio telescope in Australia. The data span is 14.2 years, from 2004 February 6 to 2018 April 25, except for PSR J0437-4715 where pre-PPTA observations from 2003 April 12 are also included. The end date of this data set corresponds to the installation and commissioning of a new ultrawide bandwidth receiver on the Parkes telescope. The observations were made typically once every 3 weeks in three radio bands $(10,20$, and $40 / 50 \mathrm{~cm})$. Details of the observing systems and data processing procedures are described in Refs. [31,32].

Pulsar times of arrival (ToAs) in PPTA DR2 are obtained using the Jet Propulsion Laboratory Solar-System ephemeris 
DE436 and the TT(BIPM18) reference time scale published by the International Bureau of Weights and Measures (BIPM). We fit these ToAs to a timing model with the standard TEMPO2 [36,37] software package. We perform the Bayesian noise analysis and search for DPDM signals using the enterprise [38] package. The PTMCMC [39] sampler is used for the stochastic sampling of parameter space and the calculation of Bayesian upper limits. We also use PyMultiNest [40] to calculate the Bayes Factor while performing model selection. The names of pulsars, basic observing information, and noise properties of the PPTA DR2 pulsars are listed in Supplemental Table S1 [34].

Timing model. The ToAs of a pulsar include quite a few terms. There are several astronomical effects that should be accounted for such as the intrinsic pulsar spin-down, the proper motion of the pulsar, the time delay due to interstellar medium, and the Shapiro delay caused by Solar-System planets. In addition to these deterministic effects, both uncorrelated noise (white noise) and correlated noise (red noise) may be present in pulsar timing data [41-46]. We model the ToAs as follows:

$$
t=t_{\mathrm{TM}}\left(\boldsymbol{\varphi}_{\mathrm{TM}}\right)+\Delta t_{\text {Noise }}\left(\boldsymbol{\vartheta}_{\text {Noise }}\right)+\Delta t_{\mathrm{DPDM}}\left(\boldsymbol{\vartheta}_{\mathrm{DPDM}}\right),
$$

where $t_{\mathrm{TM}}$ is the "timing model" that accounts for the deterministic effects, $\Delta t_{\text {Noise }}$ represents stochastic noise terms, and $\boldsymbol{\varphi}_{\mathrm{TM}}$ and $\boldsymbol{\vartheta}_{\text {Noise }}$ are parameters in the timing model and noise model, respectively. $\Delta t_{\text {DPDM }}$ is used to describe the "signal" term caused by the DPDM with paramaters $\boldsymbol{\vartheta}_{\text {DPDM }}$. We make use the TEMPO2 and enterprise software packages to determine the timing model and marginalize over model uncertainties in our Bayesian analysis. More details can be found in Refs. [24,47].

The noise term includes white noise and red noises from the rotational irregularities of the pulsar, the variations of the dispersion measure, and the band noise. The noise is assumed to follow a multivariate Gaussian distribution with a covariance matrix. For the red noises, power-law frequency dependence is assumed with parameters independently fitted for each noise term. We describe the modelings of noise in detail in the Supplemental Material [34].

The DPDM-induced ToA residuals. Within coherence region where $l<l_{c} \sim 2 \pi /\left(m_{A} v_{\text {vir }}\right)$, the DPDM can be approximately described as a plane wave, $\boldsymbol{A}(t, \boldsymbol{x})=\boldsymbol{A}_{\mathbf{0}} \sin \left(m_{A} t-\right.$ $\boldsymbol{k} \cdot \boldsymbol{x}+\boldsymbol{\alpha}(\boldsymbol{x}))$. Here $m_{A}$ is the dark photon mass, the phase term $\boldsymbol{\alpha}$ is a function of $\boldsymbol{x}[12,13]$, and $\boldsymbol{k}$ is the characteristic momentum. The direction of $\boldsymbol{k}$ is random and its magnitude is $\sim m_{A} v_{\text {vir }}$ where $v_{\text {vir }}$ is the virial velocity in our Galaxy. $\boldsymbol{A}_{\mathbf{0}}$ is the gauge potential of the DPDM background whose direction is another random vector and is unrelated to that of $\boldsymbol{k}$ in the nonrelativistic limit. The averaged value of the magnitude $\left|\boldsymbol{A}_{\mathbf{0}}\right|^{2}$ is determined by the local dark matter energy density, $2 \rho_{0} / m_{A}^{2}$, with $\rho_{0}=0.4 \mathrm{GeV} \mathrm{cm}^{-3}$ near the Earth [48]. The interference among dark photons induces a random $O(1)$ fluctuation to the magnitude. This plane-wave approximation breaks down when the measurements are performed at a time scale longer than the coherence time, $t_{c} \sim 2 \pi /\left(m_{A} v_{\text {vir }}^{2}\right)$, or at a length scale larger than the coherence length $l_{c}$. Note that we only consider the vector components of the gauge potential for dark photon field because we adopt the Lorentz gauge where the contribution from the scalar component is negligible [26].
In the Supplemental Material [34] we describe the simulations of the DPDM distribution in the local vicinity of the Solar System.

The weak coupling between the dark photon background and the "dark charge" results in an acceleration of a test body [26] as

$$
\boldsymbol{a}(t, \boldsymbol{x}) \simeq \epsilon e \frac{q}{m} m_{A} \boldsymbol{A}_{\mathbf{0}} \cos \left(m_{A} t-\boldsymbol{k} \cdot \boldsymbol{x}+\boldsymbol{\alpha}(\boldsymbol{x})\right),
$$

where $m$ is the mass of the test body, and $\epsilon$ characterizes the coupling strength of the new gauge interaction that is normalized to the electromagnetic coupling constant $e$. The dark charge $q$ equals the baryon number for the $U(1)_{B}$ interaction or baryon-minus-lepton number for the $U(1)_{B-L}$ interaction. Such an acceleration allows the detection of the DPDM in several ways, e.g., using high-precision astrometry [22] or gravitational-wave detectors [26].

The acceleration causes a displacement to a test object, which is approximately

$$
\Delta \boldsymbol{x}(t, \boldsymbol{x})=-\frac{\epsilon e q}{m m_{A}} \boldsymbol{A}_{\mathbf{0}} \cos \left(m_{A} t-\boldsymbol{k} \cdot \boldsymbol{x}+\boldsymbol{\alpha}(\boldsymbol{x})\right) .
$$

Note that here we neglect the contribution to the displacement from the spatial part $-\boldsymbol{k} \cdot \boldsymbol{x}+\boldsymbol{\alpha}(\boldsymbol{x})$. The virial velocity of dark matter is $v_{v i r} \sim 10^{-3} c$. For the dark photon mass range of interest, $10^{-23.5} \mathrm{eV} \leqslant m_{A} \leqslant 10^{-21} \mathrm{eV}$, the coherence length ranges from 0.04 to $13 \mathrm{kpc}$. For an observation of $O(10)$ years, the proper motion of a celestial body (the Sun or the pulsar) is about $3 \times 10^{-3} \mathrm{pc}$ which is much smaller than the coherence length of the DPDM field, assuming a proper motion velocity of $\sim 10^{-3} c$. The phase change for the Earth and pulsars, induced by the $-\boldsymbol{k} \cdot \boldsymbol{x}+\boldsymbol{\alpha}(\boldsymbol{x})$ term, can be safely ignored and the DPDM field can be treated as spatially uniform around the Earth or the pulsar. Therefore we can rewrite Eq. (3) as

$$
\Delta \boldsymbol{x}_{e, p}(t)=-\frac{\epsilon e q}{m m_{A}} \boldsymbol{A}_{\mathbf{0}}^{e, p} \cos \left(m_{A} t+\boldsymbol{\alpha}^{e, p}\right) ;
$$

here $\boldsymbol{A}_{\mathbf{0}}{ }^{e, p}$ and $\boldsymbol{\alpha}^{e, p}$ represent the amplitude and phase of the dark photon field at the locations of the Earth and pulsars, respectively.

Most of the pulsars studied in this work have distances between 0.1 and several $\mathrm{kpc}$, which are comparable to the coherence length. Therefore we perform the analysis in two limits:

(A) Completely uncorrelated: The phases and amplitudes of dark photon background for each pulsar are independent.

(B) Completely correlated: The phases are independent phases but with a common amplitude.

In both cases, we treat the phases of the DPDM field at each pulsar as independent free parameters because the locations to most of these pulsars are highly uncertain. Such an uncertainty results in a random phase to dark photon field characteristic to each pulsar. Note that for $m_{A}>10^{-22} \mathrm{eV}$ we only perform the uncorrelated analysis (A), since in this high-mass range most of the pulsars should lie in the uncorrelated regime (see Supplemental Fig. S2 [34]). A hybrid of correlated and uncorrelated treatment can in principle be applied based on the 
comparison between the coherence length and the separation of each pulsar pair. We expect the results of such a hybrid analysis to fall between the two cases discussed here.

We further assume a flat space time and obtain the time residual, $\Delta t_{\mathrm{DPDM}}$, induced by the DPDM

$$
\begin{aligned}
\Delta t_{\mathrm{DPDM}} & =\left(\left|\boldsymbol{d}+\Delta \boldsymbol{x}_{p}\left(t^{\prime}\right)-\Delta \boldsymbol{x}_{e}(t)\right|-|\boldsymbol{d}|\right) \\
& \simeq \boldsymbol{n} \cdot\left(\Delta \boldsymbol{x}_{p}\left(t^{\prime}\right)-\Delta \boldsymbol{x}_{e}(t)\right),
\end{aligned}
$$

where $t^{\prime}$ and $t$ are the times when the pulsar emits and the Earth receives a pulse, respectively, and $\boldsymbol{d}$ is the position vector pointing from the Earth to the pulsar, and $\boldsymbol{n}=\boldsymbol{d} /|\boldsymbol{d}|$. The timing residual caused by the DPDM is anisotropic. Specifically, the Earth term is a dipole contribution in terms of angular correlation, which is distinct from the monopole signal that derives from the gravitational effect of the general fuzzy dark matter model $[24,49]$.

Now we write the timing residuals explicitly as follows:

$$
\begin{aligned}
\Delta t_{\mathrm{DPDM}}^{(B)}= & -\frac{\epsilon e}{m_{A}}\left(\frac{q_{p}^{(B)}}{m_{p}} \boldsymbol{A}_{\mathbf{0}}^{p} \cos \left(m_{A} t+\boldsymbol{\alpha}_{p}\right)\right. \\
& \left.-\frac{q_{e}^{(B)}}{m_{e}} \boldsymbol{A}_{\mathbf{0}}^{e} \cos \left(m_{A} t+\boldsymbol{\alpha}_{e}\right)\right) \cdot \boldsymbol{n}, \\
\Delta t_{\mathrm{DPDM}}^{(B-L)}=- & \frac{\epsilon e}{m_{A}}\left(\frac{q_{p}^{(B-L)}}{m_{p}} \boldsymbol{A}_{\mathbf{0}}{ }^{p} \cos \left(m_{A} t+\boldsymbol{\alpha}_{p}\right)\right. \\
& \left.-\frac{q_{e}^{(B-L)}}{m_{e}} \boldsymbol{A}_{\mathbf{0}}{ }^{e} \cos \left(m_{A} t+\boldsymbol{\alpha}_{e}\right)\right) \cdot \boldsymbol{n},
\end{aligned}
$$

where $q_{e, p}^{(B)}, q_{e, p}^{(B-L)}, m_{e}$ and $m_{p}$ are the $B$ charge, $B-L$ charge, the mass of the Earth, and the pulsar, respectively. For $U(1)_{B}$, $q^{(B)} / m$ is approximately $1 / \mathrm{GeV}$ for both the Earth and the pulsar. For $U(1)_{B-L}, q^{(B-L)} / m$ is about $1 / \mathrm{GeV}$ for the pulsar and $0.5 / \mathrm{GeV}$ for the Earth. Note that we absorb the time difference between a pulsar and the Earth into the phase term.

Statistical analysis. We perform a likelihood-ratio test, which assesses the goodness of fit using the following statistic:

$$
\lambda_{\mathrm{LR}}=2 \ln \frac{\mathcal{L}_{\max }\left(\mathcal{H}_{1}\right)}{\mathcal{L}_{\max }\left(\mathcal{H}_{0}\right)}
$$

where $\mathcal{H}_{0}$ is the null hypothesis that the timing residuals contain only the noise contributions, and $\mathcal{H}_{1}$ is the signal hypothesis that the DPDM signal is present. In both hypotheses the noise parameters $\boldsymbol{\vartheta}_{\mathrm{W}}$ (white noise parameters), $\boldsymbol{\vartheta}_{\mathrm{SN}}$ (spin noise parameters), $\boldsymbol{\vartheta}_{\mathrm{DM}}$ (dispersion-measure noise parameters), and $\boldsymbol{\vartheta}_{\mathrm{BN}}$ (band noise parameters) are fixed at their best-fit values obtained from single pulsar analyses. The free parameters include $\boldsymbol{\vartheta}_{\mathrm{BE}}$ (Bayes ephemeris parameters vary in both $\mathcal{H}_{0}$ and $\mathcal{H}_{1}$; see Ref. [50] for details) and $\boldsymbol{\vartheta}_{\text {DPDM }}$ (DPDM parameters vary only in $\mathcal{H}_{1}$ ). The priors of all the parameters mentioned above are listed in Supplemental Table S2 [34].

We adopt the Bayesian approach to derive the constraints on the DPDM parameters. We set the upper limit on the coupling constant, $\bar{\epsilon}$, at $95 \%$ credibility as

$$
0.95=\int_{0}^{\bar{\epsilon}} P(\epsilon) d \epsilon \int \mathcal{L}\left(\epsilon, \boldsymbol{\vartheta}_{\mathrm{DPDM}}^{\prime}, \boldsymbol{\vartheta}_{\mathrm{BE}}\right) P\left(\boldsymbol{\vartheta}_{\mathrm{DPDM}}^{\prime}\right) P\left(\boldsymbol{\vartheta}_{\mathrm{BE}}\right) d \boldsymbol{\vartheta}_{\mathrm{DPDM}}^{\prime} d \boldsymbol{\vartheta}_{\mathrm{BE}}
$$

where $\vartheta_{\text {DPDM }}^{\prime}$ are the DPDM parameters excluding $m_{A}$ and $\epsilon$, and $P$ are the prior probabilities of those parameters (see Supplemental Table S2 [34]). Here the dark photon mass $m_{A}$ is singled out from $\boldsymbol{\vartheta}_{\text {DPDM }}$ as we search for possible signals for a given range of masses. In the computation of the upper limits, we fix both the white noise and the red noise parameters as their best-fit values obtained in single-pulsar analyses.

Results and discussion. Our main results about the 95\% upper limits on the coupling constant $\epsilon^{2}$, where $\epsilon$ is the dimensionless coupling constant $\epsilon$ and $\epsilon e$ is the coupling strength of the $U(1)_{B}$ or $U(1)_{B-L}$ interaction with $e$ being the electromagnetic coupling, are shown in Fig. 1. For comparison, the constraints on these parameters from experiments testing the weak equivalence principle (WEP), e.g., Ref. [20], are also presented. See the Supplemental Material [34] for a description of the WEP results. We find that our study imposes significantly improved limits in the lowmass region with $m_{A} \lesssim 3 \times 10^{-22} \mathrm{eV}$ for $U(1)_{B}$ and $m_{A} \lesssim$ $10^{-22} \mathrm{eV}$ for $U(1)_{B-L}$. Compared with the WEP results, the PPTA constraints on $\epsilon$ are stronger by one to two orders of magnitude.

We find that for a few particular frequencies, the signal hypothesis fits the PPTA DR2 data better than the null hypothesis. The strongest "signal" is at $f \simeq 1.02 \times 10^{-7} \mathrm{~Hz}$. The corresponding $m_{A}$ and $\epsilon$ are given in Supplemental Table S3 [34]. We note that the favored value of the coupling constant has already been ruled out by existing WEP experiments [20]. Therefore, it may be due to some unmodeled noise effect. A similar spurious signal also appeared in the analysis Ref. [24], which was speculated to be caused by the unmodeled perturbations of Solar System barycenter from Mercury.

In order to have a better understanding of the "signal" at $f \simeq 1.02 \times 10^{-7} \mathrm{~Hz}$, we reduce the number of pulsars and perform a consistency test. We choose two sets of pulsars: The first one includes five pulsars that contribute the most to this strongest "signal," the second one is the same except that we do not included PSR J0437-4715. Additionally, we allow red noise parameters $\boldsymbol{\vartheta}_{\mathrm{SN}}, \boldsymbol{\vartheta}_{\mathrm{DM}}$, and $\boldsymbol{\vartheta}_{\mathrm{BN}}$ to vary along with $\boldsymbol{\vartheta}_{\text {DPDM }}$ and $\boldsymbol{\vartheta}_{\mathrm{BE}}$. We calculate the Bayes factor between the null and the signal hypothesis. We find that the "signal" is mainly contributed by PSR J0437-4715. For the analysis with the other four pulsars excluding PSR J0437-4715 gives a logarithmic Bayes factor of 37 in favor of the $\mathcal{H}_{1}$ hypothesis at $f \simeq 6.58 \times 10^{-8} \mathrm{~Hz}$, which is significantly smaller than the five-pulsar analysis. The results of the tests are presented in Supplemental Table S4 [34]. Note also that the best-fit frequency differs from that of the five-pulsar analysis, and the best-fit coupling constant lies above the WEP upper limit, indicating that this may be due to unmodeled noise. More careful studies need to be carried out in the future to understand the sources of these systematics. 

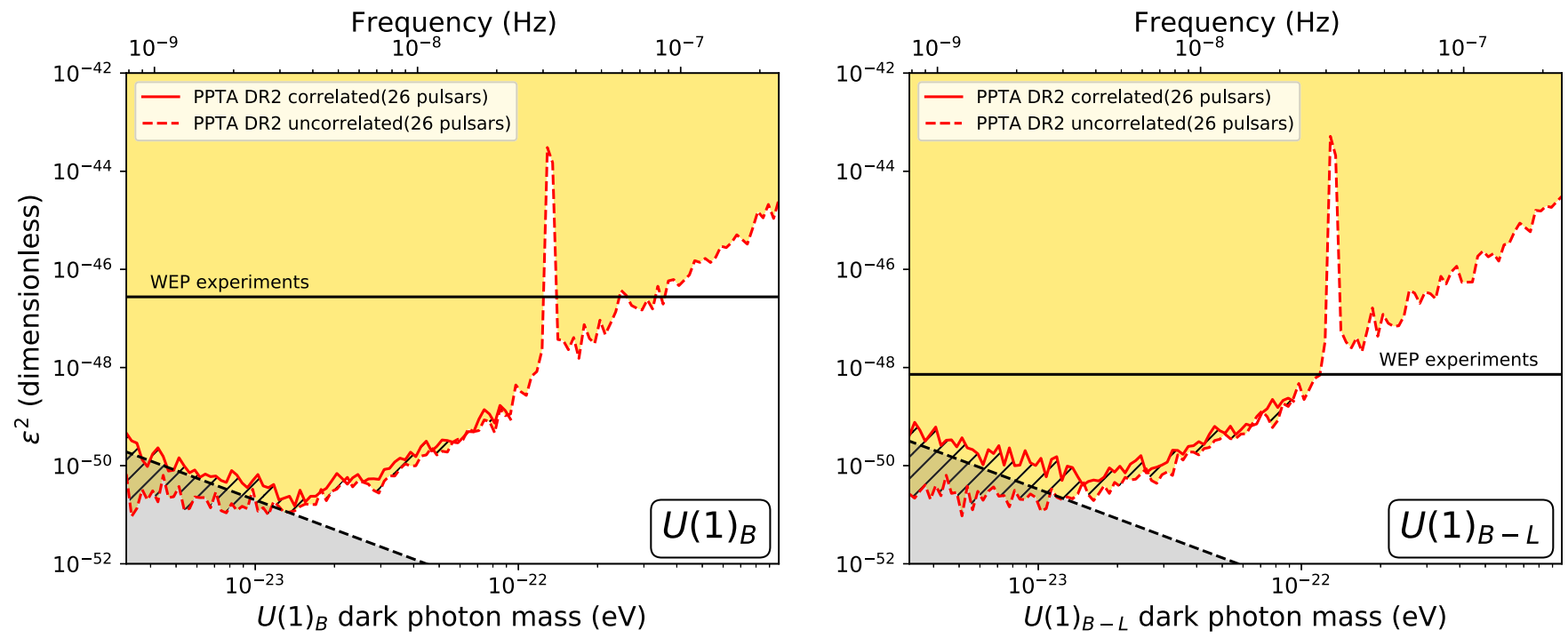

FIG. 1. Constraints on the dark photon mass $m_{A}$ and the coupling constant $\epsilon^{2}$ for the $U(1)_{B}$ (left) and $U(1)_{B-L}$ (right) gauge groups. The red solid and dashed lines are derived in this work using the PPTA DR2 data under the assumptions that the dark photon field polarizations are correlated and uncorrelated among various pulsars, respectively. The horizontal solid line is the limit from the MICROSCOPE WEP experiment [20]. The black dashed line and the gray shaded region indicate the parameter space where the gravitational effect (studied in Refs. [24,49,51]) dominates over the gauge interaction (studied here), for pulsar timing observations. More details about the WEP experiments and gravitational effects are presented in the Supplemental Material [34]. Note that in the WEP experiments, the dark photon is not required to be the dark matter.

We note that the mass range with the strongest constraint is below the typical fuzzy dark matter mass $\left(10^{-22} \mathrm{eV}\right)$. Currently there is no consensus on the constraints on the mass of the fuzzy dark matter. The stellar kinematics of dwarf spheroidal galaxies tend to favor a lower mass [52,53] (a few times of $10^{-23} \mathrm{eV}$ ), while the halo mass function derived from the Lyman- $\alpha$ forest suggests a higher lower limit $[16,23]$ $\left(\gtrsim 10^{21} \mathrm{eV}\right)$. Most of these constraints rely on assumptions about the structure formation in the fuzzy dark matter scenario. More detailed studies of the interplay between the fuzzy dark matter and the baryonic matter, such as the dark matter-to-baryon mass ratio or various baryonic effects in fuzzy dark matter simulations, are required to reach a robust and consistent result.

The gray shaded regions in Fig. 1 indicate the parameter space for which the "gravitational effect" due to the space-time metric oscillation induced by the wavelike DPDM field dominates over the gauge interaction discussed in this work. See the Supplemental Material [34] for more details of the estimate of the gray regions. In such parameter regions, dedicated analysis with both effects being included in the signal model is required and will be studied in future work.

Summary. Ultralight fuzzy dark matter is proposed as an attractive candidate of dark matter in the universe which helps solve the small-scale crises of the classical cold dark matter scenario. Using the precise timing observations of 26 pulsars by the PPTA project, we study the possible couplings between ultralight dark matter and ordinary matter. Taking the DPDM as an example, we obtain by far the strongest constraints on the parameters of the DPDM. The upper limits on the dimensionless coupling constant $\epsilon$ derived in our study are improved by up to two orders of magnitude when the dark photon mass is smaller than $3 \times 10^{-22} \mathrm{eV}\left(10^{-22} \mathrm{eV}\right)$ for the $U(1)_{B}\left(U(1)_{B-L}\right)$ scenario.

The search sensitivity for the DPDM is expected to improve significantly in the near future as more pulsars are monitored with continually extending data spans by the worldwide PTA campaigns including the PPTA, the North American Nanohertz Observatory for Gravitational Waves (NANOGrav [54]), and the European PTA (EPTA [55]), which have jointly formed the International PTA (IPTA [56]). The Five-hundred-meter Aperture Spherical Telescope (FAST [57]) and the Square Kilometer Array (SKA [58]) are also expected to join the IPTA collaboration. These efforts are likely to bring revolutionary progress in studying a wide range of dark matter models and more generally in answering the related fundamental questions in physics.

Acknowledgments. This work is supported by the National Natural Science Foundation of China (No. 11722328, No. 11851305, No. 12003069 , No. 11947302 , No. 11690022 , No. 11851302, No. 11675243, and No. 11761141011). X.X. is supported by Deutsche Forschungsgemeinschaft under Germany's Excellence Strategy EXC2121 "Quantum Universe" 390833306. Q.Y. is supported by the Key Research Program of the Chinese Academy of Sciences (No. XDPB15) and the Program for Innovative Talents and Entrepreneur in Jiangsu. J.S. is supported by the Strategic Priority Research Program and Key Research Program of Frontier Science of the Chinese Academy of Sciences under Grants No. XDB21010200, No. XDB23010000, and No. ZDBS-LY-7003 and CAS project for Young Scientists in Basic Research YSBR-006. X.J.Z., A.D.C., B.G., D.J.R., and R.M.S. are 
supported by ARC CE170100004. Z.Q.X. is supported by the Entrepreneurship and Innovation Program of Jiangsu Province. Y.Z. is supported by U.S. Department of Energy under Award Number DESC0009959. R.M.S. is the recipient of ARC Future Fellowship FT190100155. J.W. is supported by the Youth Innovation Promotion Association of
Chinese Academy of Sciences. S.D. is the recipient of an Australian Research Council Discovery Early Career Award (DE210101738) funded by the Australian Government. The Parkes radio telescope is part of the Australia Telescope, which is funded by the Commonwealth Government for operation as a National Facility managed by CSIRO.
[1] N. A. Bahcall, J. P. Ostriker, S. Perlmutter, and P. J. Steinhardt, The cosmic triangle: Revealing the state of the universe, Science 284, 1481 (1999).

[2] D. H. Weinberg, J. S. Bullock, F. Governato, R. K. de Naray, and A. H. G. Peter, Cold dark matter: Controversies on small scales, Proc. Nat. Acad. Sci. U.S.A. 112, 12249 (2015).

[3] T. K. Chan, D. Kereš, J. Onorbe, P. F. Hopkins, A. L. Muratov, C.-A. Faucher-Giguere, and E. Quataert, The impact of baryonic physics on the structure of dark matter haloes: The view from the FIRE cosmological simulations, Mon. Not. R. Astron. Soc. 454, 2981 (2015).

[4] P. Bode, J. P. Ostriker, and N. Turok, Halo formation in warm dark matter models, Astrophys. J. 556, 93 (2001).

[5] S. Tulin and H. B. Yu, Dark matter self-interactions and small scale structure, Phys. Rept. 730, 1 (2018).

[6] W. Hu, R. Barkana, and A. Gruzinov, Fuzzy Cold Dark Matter: The Wave Properties of Ultralight Particles, Phys. Rev. Lett. 85, 1158 (2000).

[7] C. P. Burgess, J. P. Conlon, L.-Y. Hung, C. H. Kom, A. Maharana, and F. Quevedo, Continuous global symmetries and hyperweak interactions in string compactifications, J. High Energy Phys. 07 (2008) 073.

[8] M. Goodsell, J. Jaeckel, J. Redondo, and A. Ringwald, Naturally light hidden photons in LARGE volume string compactifications, J. High Energy Phys. 11 (2009) 027.

[9] M. Cicoli, M. Goodsell, J. Jaeckel, and A. Ringwald, Testing string vacua in the lab: From a hidden CMB to dark forces in flux compactifications, J. High Energy Phys. 07 (2011) 114.

[10] In order to give a mass to the dark photon, a Higgs boson in the dark section may be introduced. Since such a Higgs is also very weakly coupled to the Standard Model sector, its existence is challenging to be tested. Furthermore, if one pushes the dark Higgs coupling to be very small, takes its vacuum expectation value to be very large, meanwhile maintains the product of these two parameters to be finite, we reach the Stueckelberg limit where the dark Higgs is very heavy and effectively decoupled from the rest of the theory, except the dark photon mass it generates.

[11] The occupation number is $\rho_{0} / m_{A} \sim 10^{30} \mathrm{~cm}^{-3}$, where $\rho_{0}$ is the local dark matter energy density.

[12] H.-Y. Schive, T. Chiueh, and T. Broadhurst, Cosmic structure as the quantum interference of a coherent dark wave, Nat. Phys. 10, 496 (2014).

[13] H.-Y. Schive, M.-H. Liao, T.-P. Woo, S.-K. Wong, T. Chiueh, T. Broadhurst, and W. Y. P. Hwang, Understanding the Core-Halo Relation of Quantum Wave Dark Matter from 3D Simulations, Phys. Rev. Lett. 113, 261302 (2014).

[14] J. Zhang, Y.-L. S. Tsai, J.-L. Kuo, K. Cheung, and M.-C. Chu, Ultralight axion dark matter and its impact on dark halo structure in N-body simulations, Astrophys. J. 853, 51 (2018).
[15] J. F. Navarro, C. S. Frenk, and S. D. M. White, A universal density profile from hierarchical clustering, Astrophys. J. 490, 493 (1997).

[16] L. Hui, J. P. Ostriker, S. Tremaine, and E. Witten, Ultralight scalars as cosmological dark matter, Phys. Rev. D 95, 043541 (2017).

[17] X. Cui, A. Abdukerim, W. Chen, X. Chen, Y. Chen, B. Dong, D. Fang, C. Fu, K. Giboni, F. Giuliani et al. (PandaX-II Collaboration), Dark Matter Results from 54-Ton-Day Exposure of PandaX-II Experiment, Phys. Rev. Lett. 119, 181302 (2017).

[18] D. S. Akerib, S. Alsum, H. M. Araújo, X. Bai, A. J. Bailey, J. Balajthy, P. Beltrame, E. P. Bernard, A. Bernstein, T. P. Biesiadzinski et al. (LUX Collaboration), Results from a Search for Dark Matter in the Complete LUX Exposure, Phys. Rev. Lett. 118, 021303 (2017).

[19] E. Aprile, J. Aalbers, F. Agostini, M. Alfonsi, L. Althueser, F. D. Amaro, M. Anthony, F. Arneodo, L. Baudis, B. Bauermeister et al. (XENON Collaboration), Dark Matter Search Results from a One Ton-Year Exposure of XENON1T, Phys. Rev. Lett. 121, 111302 (2018).

[20] J. Bergé, P. Brax, G. Métris, M. Pernot-Borràs, P. Touboul, and J.-P. Uzan, MICROSCOPE Mission: First Constraints on the Violation of the Weak Equivalence Principle by a Light Scalar Dilaton, Phys. Rev. Lett. 120, 141101 (2018).

[21] P. W. Graham, D. E. Kaplan, J. Mardon, S. Rajendran, W. A. Terrano, Dark matter direct detection with accelerometers, Phys. Rev. D 93, 075029 (2016).

[22] H.-K. Guo, Y. Ma, J. Shu, X. Xue, Q. Yuan, and Y. Zhao, Detecting dark photon dark matter with Gaia-like astrometry observations, J. Cosmol. Astropart. Phys. 05 (2019) 015.

[23] E. Armengaud, N. Palanque-Delabrouille, C. Yeche, D. J. E. Marsh, and J. Baur, Constraining the mass of light bosonic dark matter using SDSS Lyman- $\alpha$ forest, Mon. Not. R. Astron. Soc. 471, 4606 (2017).

[24] N. K. Porayko, X. Zhu, Y. Levin, L. Hui, G. Hobbs, A. Grudskaya, K. Postnov, M. Bailes, N. D. R. Bhat, W. Coles et al. (PPTA Collaboration), Parkes pulsar timing array constraints on ultralight scalar-field dark matter, Phys. Rev. D 98, 102002 (2018).

[25] I. De Martino, T. Broadhurst, S.-H. H. Tye, T. Chiueh, H.-Y. Schive, and R. Lazkoz, Recognizing Axionic Dark Matter by Compton and De Broglie Scale Modulation of Pulsar Timing, Phys. Rev. Lett. 119, 221103 (2017).

[26] A. Pierce, K. Riles, and Y. Zhao, Searching for Dark Photon Dark Matter with Gravitational-Wave Detectors, Phys. Rev. Lett. 121, 061102 (2018).

[27] H.-K. Guo, K. Riles, F.-W. Yang, and Y. Zhao, Searching for dark photon dark matter in LIGO O1 data, Commun. Phys. 2, 155 (2019).

[28] R. Abbott, T. D. Abbott, F. Acernese, K. Ackley, C. Adams, N. Adhikari, R. X. Adhikari, V. B. Adya, C. Affeldt, D. Agarwal 
et al. (LIGO Scientific, Virgo and KAGRA), Constraints on dark photon dark matter using data from LIGO's and Virgo's third observing run, arXiv:2105.13085.

[29] Recently, the NANOGrav team claimed a detection of a common-spectrum stochastic process in their 12.5-yr data set [30]. The NANOGrav 'feature,' which is modeled as a powerlaw spectrum in the low-frequency range, is different from the monochromatic signal induced by the DPDM studied in this work.

[30] Z. Arzoumanian, P. T. Baker, H. Blumer, B. Becsy, A. Brazier, P. R. Brook, S. Burke-Spolaor, S. Chatterjee, S. Chen, J. M. Cordes et al. (NANOGrav collaboration), The NANOGrav 12.5 yr data set: Search for an isotropic stochastic gravitational-wave background, Astrophys. J. Lett. 905, L34 (2020).

[31] R. N. Manchester, G. Hobbs, M. Bailes, W. A. Coles, W. van Straten, M. J. Keith, R. M. Shannon, N. D. R. Bhat, A. Brown, S. G. Burke-Spolaor et al., The Parkes Pulsar Timing Array Project, Publ. Astron. Soc. Aust. 30, e017 (2013).

[32] M. Kerr, D. J. Reardon, G. Hobbs, R. M. Shannon, R. N. Manchester, S. Dai, C. J. Russell, S.-B. Zhang, W. van Straten, S. Osłowski et al., The Parkes Pulsar Timing Array Project: Second data release, Publ. Astron. Soc. Aust. 37, e020 (2020).

[33] R. M. Shannon, V. Ravi, L. T. Lentati, P. D. Lasky, G. Hobbs, M. Kerr, R. N. Manchester, W. A. Coles, Y. Levin, M. Bailes et al., Gravitational waves from binary supermassive black holes missing in pulsar observations, Science 349, 1522 (2015).

[34] See Supplemental Material at http://link.aps.org/supplemental/ 10.1103/PhysRevResearch.4.L012022 for the properties of the pulsar used in the analyses, as well as the details of the noise model, the statistical properties of the DPDM field, the parameter setting for the Bayesian analyses, and results of the Bayesian search.

[35] https://doi.org/10.25919/5db90a8bdeb59

[36] G. B. Hobbs, R. T. Edwards, and R. N. Manchester, TEMPO2, a new pulsar-timing package-I. An overview, Mon. Not. R. Astron. Soc. 369, 655 (2006).

[37] R. T. Edwards, G. B. Hobbs, and R. N. Manchester, TEMPO2, a new pulsar timing package-II. The timing model and precision estimates, Mon. Not. R. Astron. Soc. 372, 1549 (2006).

[38] J. A. Ellis, M. Vallisneri, S. R. Taylor, and P. T. Baker, ENTERPRISE: Enhanced Numerical Toolbox Enabling a Robust PulsaR Inference SuitE, Zenodo (2020), doi: 10.5281/zenodo.4059815.

[39] J. Ellis and R. van Haasteren, jellis18/PTMCMCSampler: Official Release, Zenodo (2017), doi: 10.5281/zenodo.1037579.

[40] J. Buchner, A. Georgakakis, K. Nandra, L. Hsu, C. Rangel, M. Brightman, A. Merloni, M. Salvato, J. Donley, and D. Kocevski, X-ray spectral modelling of the AGN obscuring region in the CDFS: Bayesian model selection and catalogue, Astron. Astrophys. 564, A125 (2014).

[41] R. van Haasteren and Y. Levin, Understanding and analysing time-correlated stochastic signals in pulsar timing, Mon. Not. R. Astron. Soc. 428, 1147 (2012).

[42] R. van Haasteren and M. Vallisneri, Low-rank approximations for large stationary covariance matrices, as used in the Bayesian and generalized-least-squares analysis of pulsar-timing data, Mon. Not. R. Astron. Soc. 446, 1170 (2014).
[43] R. N. Caballero, K. J. Lee, L. Lentati, G. Desvignes, D. J. Champion, J. P. W. Verbiest, G. H. Janssen, B. W. Stappers, M. Kramer, P. Lazarus et al., The noise properties of 42 millisecond pulsars from the European Pulsar Timing Array and their impact on gravitational-wave searches, Mon. Not. R. Astron. Soc. 457, 4421 (2016).

[44] L. Lentati, R. M. Shannon, W. A. Coles, J. P. W. Verbiest, R. van Haasteren, J. A. Ellis, R. N. Caballero, R. N. Manchester, Z. Arzoumanian, S. Babak et al., From spin noise to systematics: Stochastic processes in the first International Pulsar Timing Array data release, Mon. Not. R. Astron. Soc. 458, 2161 (2016).

[45] X. P. You, G. Hobbs, W. A. Coles, R. N. Manchester, R. Edwards, M. Bailes, J. Sarkissian, J. P. W. Verbiest, W. Van Straten, A. Hotan et al., Dispersion measure variations and their effect on precision pulsar timing, Mon. Not. R. Astron. Soc. 378, 493 (2007).

[46] M. J. Keith, W. Coles, R. M. Shannon, G. B. Hobbs, R. N. Manchester, M. Bailes, N. D. R. Bhat, S. Burke-Spolaor, D. J. Champion, A. Chaudhary et al., Measurement and correction of variations in interstellar dispersion in high-precision pulsar timing, Mon. Not. R. Astron. Soc. 429, 2161 (2012).

[47] L. Lentati, P. Alexander, M. P. Hobson, F. Feroz, R. van Haasteren, K. J. Lee, and R. M. Shannon, TEMPONEST: A Bayesian approach to pulsar timing analysis, Mon. Not. R. Astron. Soc. 437, 3004 (2013).

[48] R. Catena and P. Ullio, A novel determination of the local dark matter density, J. Cosmol. Astropart. Phys. 08 (2010) 004.

[49] A. Khmelnitsky and V. Rubakov, Pulsar timing signal from ultralight scalar dark matter, J. Cosmol. Astropart. Phys. 02 (2014) 019.

[50] Z. Arzoumanian, P. T. Baker, A. Brazier, S. Burke-Spolaor, S. J. Chamberlin, S. Chatterjee, B. Christy, J. M. Cordes, N. J. Cornish, F. Crawford et al., The NANOGrav 11-year data set: Pulsar-timing constraints on the stochastic gravitational-wave background, Astrophys. J. 859, 47 (2018).

[51] K. Nomura, A. Ito, and J. Soda, Pulsar timing residual induced by ultralight vector dark matter, Eur. Phys. J. C 80, 419 (2020).

[52] A. X. Gonzaláz-Morales, D. J. E. Marsh, J. Peñarrubia, and L. A. Ureña-López, Unbiased constraints on ultralight axion mass from dwarf spheroidal galaxies, Mon. Not. R. Astron. Soc. 472, 1346 (2017).

[53] E. Kendal and R. Easther, The Core-Cusp problem revisited: ULDM vs. CDM, Publ. Astron. Soc. Austral. 37, e009 (2020).

[54] M. A. McLaughlin, The North American Nanohertz Observatory for gravitational waves, Class. Quant. Grav. 30, 224008 (2013).

[55] M. Kramer and D. J. Champion, The European pulsar timing array and the large European array for pulsars, Class. Quant. Grav. 30, 224009 (2013).

[56] R. N. Manchester, The International Pulsar Timing Array, Class. Quant. Grav. 30, 224010 (2013).

[57] R. Nan, D. Li, C. Jin, Q. Wang, L. Zhu, W. Zhu, H. Zhang, Y. Yue, and L. Qian, The Five-Hundred-Meter Aperture Spherical Radio Telescope (FAST) Project, Int. J. Mod. Phys. D 20, 989 (2011).

[58] T. J. W. Lazio, The square kilometre array Pulsar Timing Array, Class. Quant. Grav. 30, 224011 (2013). 